\title{
Lidil
}

Revue de linguistique et de didactique des langues

Acquisition et enseignement de la morphographie

\section{La forme contre l'usage}

Étude exploratoire de l'acquisition du prétérit anglais par des

apprenants français

Coralie Payre-Ficout et Jean-Pierre Chevrot

\section{(2) OpenEdition}

Édition électronique

URL : http://journals.openedition.org/lidil/813

DOI : $10.4000 /$ lidil. 813

ISSN : 1960-6052

Éditeur

UGA Éditions/Université Grenoble Alpes

\section{Édition imprimée}

Date de publication : 1 décembre 2004

Pagination : 101-115

ISBN : 2-914176-11-2

ISSN : $1146-6480$

\section{Référence électronique}

Coralie Payre-Ficout et Jean-Pierre Chevrot, "La forme contre l'usage », Lidil [En ligne], 30 | 2004, mis en ligne le 29 janvier 2008, consulté le 19 avril 2019. URL : http://journals.openedition.org/lidil/813 ;

DOI : 10.4000/lidil.813 


\title{
LA FORME CONTRE L'USAGE
}

\author{
ÉTUDE EXPLORATOIRE DE L'ACQUISITION DU PRÉTÉRIT
}

ANGLAIS PAR DES APPRENANTS FRANÇAIS

Coralie PAYRE-FICOUT et Jean-Pierre CHEVROT *

Dans de nombreuses langues, l'apprentissage et la mise en œuvre de l'orthographe grammaticale restent des problèmes majeurs, auxquels se trouvent confrontés les scripteurs enfants ou adultes. Dans le cas de l'orthographe du français, les erreurs sur l'accord en nombre des formes verbales écrites en $/ E /$ (-ai(en)t, -é(es)) demeurent fréquentes au moins jusqu'à la classe de troisième (Brissaud et Chevrot, 2000). Plus généralement, des erreurs affectant la morphologie verbale subsistent même dans les écrits d'adultes de niveau de formation élevé (Lucci et Millet, 1994).

Les nombreuses étapes que doivent franchir les jeunes apprenants francophones ou anglophones pour résoudre les difficultés grammaticales de l'orthographe de leur propre langue commencent à être connues (Thévenin, Totereau, Fayol, 1997; Nunes, Bryant et Bindman, 1997). En revanche, on ignore presque tout de ce processus quand il s'agit de l'apprentissage de la morphologie d'une langue étrangère enseignée dans le cadre scolaire. L'acquisition du prétérit oral entre 2 et 6 ans et l'apprentissage de sa forme écrite ayant été largement décrits chez les jeunes anglophones, le choix de ce

* Université Stendhal-Grenoble 3, Laboratoire Lidilem. 
temps garantit la comparaison entre les processus d'apprentissage en langue maternelle et en langue étrangère.

En guise d'introduction, nous commencerons par présenter les principaux résultats concernant l'apprentissage du prétérit anglais par de jeunes Anglais, puis nous essayerons de cerner les conditions spécifiques caractérisant l'apprentissage de la morphologie verbale dans le cadre scolaire. Ensuite, nous proposerons une analyse contrastive des temps du passé en anglais et en français afin d'observer les secteurs de complexité potentielle pour les apprenants francophones. Enfin, nous présenterons une étude exploratoire de l'acquisition du prétérit anglais dans des textes écrits par des apprenants francophones. Nous la complèterons par une expérimentation construite à partir des hypothèses déduites de l'observation des formes verbales dans ces textes. Les résultats convergent pour suggérer que la conscience morphosyntaxique des apprenants, c'est-à-dire leur capacité à prendre conscience des unités morphosyntaxiques et à les manipuler délibérément, se centre sur la forme des verbes français au détriment des conditions d'usage du prétérit en anglais.

\section{Apprentissage du prétérit par les jeunes Anglais}

Terezinha Nunes et ses collègues (1997) ont étudié l'apprentissage de i'ortínographe du prétérit anglais en se focalisant sur les terminaisons - $e d$. Selon leur description, le développement orthographique se déroulerait en cinq étapes. Lors de la première étape, dénommée étape pré-phonétique, les enfants n'orthographient pas systématiquement la terminaison des mots et donnent la base verbale. La seconde étape commence lorsque les enfants orthographient phonétiquement les terminaisons mais ne prêtent pas attention aux distinctions grammaticales. Ainsi, kissed devient kist. Lors de la troisième étape dite intermédiaire, les enfants devenus conscients que les correspondances lettre-son sont parfois inappropriées commencent à utiliser la terminaison -ed. Cette étape se caractérise par la prise de conscience que certains mots se terminant par le son/d/, /t/, /id/ s'orthographient -ed. Cependant les enfants ne saisissent pas la signification grammaticale de 
la graphie -ed. Ainsi, ils tendent à associer cette orthographe ed à des verbes mais également à d'autres catégories lexicales, par exemple des adjectifs (soft devenant sofed). La reconnaissance d'un lien entre grammaire et orthographe est la caractéristique de la quatrième étape. Durant cette étape les enfants utilisent la terminaison -ed pour les verbes du passé mais ils la généralisent aussi aux verbes irréguliers. Enfin, lors de la cinquième étape les enfants écrivent -ed uniquement à la fin des verbes réguliers. L'étude de Nunes et al. (1997) suggère que l'évolution dans l'utilisation du patron orthographique -ed est étroitement liée à la conscience grammaticale: plus celle-ci est développée plus les enfants utilisent rapidement -ed de manière appropriée.

\section{Spécificités de l'apprentissage de la morphologie verbale en contexte scolaire}

Les conditions dans lesquelles les écoliers anglophones et les collégiens francophones abordent l'orthographe du verbe anglais sont différentes. Premièrement, les collégiens francophones sont plus âgés et disposent d'une maturation cognitive et d'un savoir métalinguistique sur leur langue maternelle qui modifient les conditions de la construction du savoir en langue étrangère. Deuxièmement, quand ils abordent I'apprentissage de l'orthographe, les écoliers anglais connaissent déjà les formes orales des verbes de leur langue. Pour eux, l'apprentissage orthographique se limite donc à apprendre une forme écrite face à une forme orale déjà maitrisée. De leur côté, les francophones doivent apprendre simultanément la forme orale et sa graphie. Troisièmement, lorsque les jeunes Anglais ont appris la morphologie verbale à l'oral, entre 2 et 6 ans, ils ont dû découvrir par eux-mêmes les régularités et les irrégularités. De leur côté, les collégiens francophones qui apprennent la conjugaison de l'anglais bénéficient d'un enseignement explicite des règles de formation et d'usage. S'agissant du prétérit, les enseignants donnent en effet la règle «ajouter -ed» aux collégiens francophones, qui apprennent par ailleurs les verbes irréguliers par cœur sous forme de listes. 
La connaissance préalable d'une langue peut favoriser mais également gêner la construction des savoirs en langue étrangère. L'analyse contrastive des systèmes linguistiques permet de pointer les zones de difficultés potentielles.

\section{Analyse contrastive des temps du passé en anglais et en français}

S'agissant des temps du passé, l'anglais et le français sont différents au niveau de l'organisation des paradigmes et de leur usage.

Les deux systèmes d'aspect et de temps ne sont pas superposables. Si l'on considère par exemple la notion de passé, on est loin d'une correspondance terme à terme entre les paradigmes des deux langues. Selon le contexte, au prétérit anglais peut correspondre l'usage du passé composé, de l'imparfait et du passé simple. Au present perfect, correspondent le passé composé et le présent. Si l'on part du français, comme le font sans doute les apprenants débutants de l'anglais, les correspondances sont aussi complexes. En face du passé composé, il faudra choisir entre un prétérit et un present perfect. Seul le passé simple renvoie de façon univoque au prétérit.

Sur le plan de la forme, la langue française est une langue fíexionnelie riche, notamment à l'écrit, caractérisée par un décalage entre la morphologie de l'oral et celle de l'écrit (Jaffré, 1998). Plus précisément, l'écrit distingue des formes verbales qui sont homophones à l'oral. La connaissance de la morphologie verbale orale ne permet donc pas de prédire les formes écrites, la transcription phonographique directe de l'oral ne prenant en compte ni l'existence de morphogrammes muets ni la multiplicité des liens entre phonèmes et graphèmes (dans le cas des formes verbales homophones en $/ F /$ par exemple). Ainsi, la grammaire joue un rôle primordial dans la sélection de la flexion écrite. À l'inverse du français, l'anglais présente un système flexionnel extrêmement pauvre. La langue anglaise ne compte que deux temps grammaticaux, le présent et le passé, et ne connait, à l'exception de be et de quelques verbes irréguliers, que trois marqueurs de la forme 
simple, deux pour le présent simple ( $-s$ et $\varnothing$ ) et un seul pour le prétérit $(-e d)$. On peut trouver un verbe anglais sous quatre formes s'il est régulier (play, plays, played, playing) et entre trois et cinq s'il est irrégulier (Lapaire et Rotgé, 1991; Larreya et Rivière, 1991). À l'inverse du français, la graphie des verbes anglais neutralise souvent des différences sonores (cf. les différentes prononciations de la terminaison -ed). De ce fait, à la différence du français, toute flexion écrite de l'anglais a un correspondant oral, ce qui facilite la scription. Toutefois, à une flexion écrite unique peuvent correspondre différentes prononciations. Comme en français, la transcription phonographique ne garantit donc pas la justesse morphographique.

En définitive, dans les deux langues, les apprenants doivent apprendre à se détacher de la forme orale pour produire la forme écrite, mais ils procèdent de manière différente. Les apprenants de la morphographie du français doivent plutôt comprendre qu'une même séquence sonore peut s'écrire de façons différentes. À l'inverse, les apprenants de la morphographie de l'anglais doivent plutôt comprendre que des séquences sonores différentes peuvent s'écrire de la même façon.

Ces différences dans l'usage et la forme des temps passés des deux langues devraient se manifester par des erreurs concernant ces deux plans dans les textes des jeunes Français apprenant l'anglais.

Étude exploratoire du prétérit dans les textes de jeunes Français

Nous avons réalisé une étude exploratoire auprès de 107 apprenants, répartis sur cinq niveaux différents: classes de quatrième $(\mathrm{N}=20)$ et troisième $(\mathrm{N}=20)$ du collège, classes de seconde $(\mathrm{N}=30)$ et première $(\mathrm{N}=23)$ du lycée et étudiants de deuxième année de deug Langues, Littératures et Civilisations Etrangères, spécialité anglais $(\mathrm{N}=14)$. Nos objectifs étaient d'élaborer une typologie d'erreurs, d'esquisser le patron d'acquisition des apprenants français et de le comparer à celui des jeunes Anglais. 
La tâche consistait à demander aux sujets de réaliser une production libre avec la consigne implicite d'utiliser le prétérit (narration d'un événement révolu). Dans une première étape, nous avons analysé les formes verbales justes. Cette analyse montre que globalement les élèves réussissent plutôt bien à former le prétérit $(68,4 \%$ de réussite). De façon inattendue, ce taux de réussite est significativement ${ }^{1}$ plus élevé pour les verbes irréguliers $(77,2 \%)$ que pour les verbes réguliers $(68,8 \%)$, même si nous ne retrouvons pas cette différence à l'intérieur de chaque niveau scolaire. Si l'on observe l'évolution de la réussite en fonction des niveaux, on constate que les taux stagnent en quatrième et troisième, puis marquent une progression significative lors du passage en seconde et jusqu'à l'université en deuxième année de deug.

Dans une deuxième étape, nous avons classé les 334 erreurs en dix catégories majeures dont certaines se divisent en sous catégories. La définition de ces catégories est donnée dans le Tableau 1. Dans le Tableau 2 apparaissent les fréquences d'occurrence des catégories et des sous-types, classés par ordre décroissant des incidences.

\section{Erreurs d'irrégularisation/régularisation}

Erreurs de régularisation (adjonction du suffixe - $e d$ à des verbes irréguliers : taked au lieu de took) et erreurs d'irrégularisation (la forme verbale du prétérit est construite par analogie avec un verbe irrégulier: toke au lieu de took par analogie à break qui fait broke).

\section{Création de formes verbales composées erronées}

Formes verbales qui ont été probablement calquées sur les temps composés du français: erreurs Have conjugué (have see au lieu de saw); erreurs Be conjugué (are sing au lieu de sang).

\section{Erreurs de modification orthographique}

Formes dont la base n'est pas conforme à une règle de modification orthographique dépendant de la morphologie; comprennent les modifications orthographiques entraînées par -ing (was sking au lieu de was skiing) et les modifications orthographiques entraînées par -ed (cryed au lieu de cried).

1. Nous avons appliqué, quand c'était possible, des tests d'inférence statistique (Wilcoxon, U de Man-Whitney, Kruskal-Wallis, Chi 2). Nous ne rentrerons pas ici dans le détail de ces tests, dont nous ne donnerons que la sortie (significatif ou non significatif). 


\section{Formes non prédicatives}

Formes simples qui existent en anglais mais qui ne fonctionnent pas seules comme prédicat d'une phrase ; remplacement du prétérit par une forme en -ing (playing au lieu de played) ou par un participe passé.

\section{Formes négatives}

Non-respect des règles de construction de l'auxiliaire du présent simple do ou de l'auxiliaire du prétérit simple did à la forme négative (don't knew au lieu de didn't know).

Erreurs d'accord sur be

Erreurs d'accord en nombre sur l'auxiliaire be (was remplace were).

\section{Erreurs par substitution de formes existantes}

Remplacement du prétérit simple par une autre forme verbale existante en anglais : présent simple ou base verbale, présent progressif, prétérit progressif, present perfect, past perfect.

\section{Erreurs d'orthographe}

Formes verbales dont la base comporte une erreur qui ne peut pas être considérée comme une irrégularisation : omission (wen remplace went), adjonction, substitution, écriture phonétique.

\section{Erreurs de sens}

Emploi d'un verbe anglais à la place d'un autre (read au lieu de watch).

\section{Erreurs d'emprunt}

Formes verbales qui n'existent pas en anglais et qui sont formées par analogie avec des verbes français (ported au lieu de carried).

Tableau 1-Classification des erreurs

Après avoir retiré du corpus les erreurs ne relevant pas de la morphologie verbale (catégories $6,12,14,15,17 \mathrm{du}$ Tableau 2, p. suiv.), nous avons constaté que sur les 284 erreurs restantes, 142 consistaient à produire une forme anglaise composée, existante ou non, à la place du prétérit simple attendu (catégories $3,4,5,8,9,10,16,18$ ). Ces résultats suggèrent une nette influence des temps composés du français sur le choix de la forme anglaise.

Afin d'étayer cette hypothèse, nous avons observé si dans les formes composées erronées, les auxiliaires utilisés à tort correspondaient aux auxiliaires être et avoir du français.

Dans le Tableau 3, on constate que les élèves utilisent massivement en anglais l'auxiliaire exigé par le verbe français au passé composé (69\%). Par exemple, en troisième, $84 \%$ des formes composées erronées sont de type he is get up, 


\begin{tabular}{|c|c|c|c|}
\hline Type erreur & Nb & $\%$ & Exemple \\
\hline $\begin{array}{l}1 \text { - Erreur d'usage : } \\
\text { présent remplace prétérit }\end{array}$ & 69 & $21^{\circ}$ & $\begin{array}{l}\text { fear remplace } \\
\text { feared }\end{array}$ \\
\hline $\begin{array}{l}2 \text { - Forme non } \\
\text { prédicative }\end{array}$ & 35 & 10 & $\begin{array}{l}\text { sleeping remplace } \\
\text { slept }\end{array}$ \\
\hline 3 - Be conjugué prétérit & 33 & 10 & was eat \\
\hline 4 - Be conjugué présent & 24 & 7 & are sing \\
\hline $\begin{array}{l}5 \text { - Erreur d'usage : } \\
\text { prétérit progressif } \\
\text { remplace prétérit }\end{array}$ & 24 & 7 & $\begin{array}{l}\text { was walking } \\
\text { remplace walked }\end{array}$ \\
\hline 6 - Erreur de sens & 19 & 6 & do remplace go \\
\hline 7 -Régularisation & 18 & 5 & $\begin{array}{l}\text { taked remplace } \\
\text { took }\end{array}$ \\
\hline $\begin{array}{l}8 \text { - Have conjugué } \\
\text { présent }\end{array}$ & 18 & 5 & $\begin{array}{l}\text { have see } \\
\text { remplace saw }\end{array}$ \\
\hline $\begin{array}{l}9 \text { - Erreur d'usage : } \\
\text { present perfect remplace } \\
\text { prétérit }\end{array}$ & 18 & 5 & $\begin{array}{l}\text { have skied } \\
\text { remplace skied }\end{array}$ \\
\hline $\begin{array}{l}10 \text { - Have conjugué } \\
\text { prétérit }\end{array}$ & 13 & 4 & $\begin{array}{l}\text { had see remplace } \\
\text { saw }\end{array}$ \\
\hline $\begin{array}{l}11 \text { - Erreur de } \\
\text { modi fication } \\
\text { orthographique }\end{array}$ & 11 & 3 & $\begin{array}{l}\text { was sking } \\
\text { remplace was } \\
\text { skiing }\end{array}$ \\
\hline 12 - Erreur d'orthographe & 11 & 3 & $\begin{array}{l}\text { wen remplace } \\
\text { went }\end{array}$ \\
\hline 13 - Irrégulari sation & 9 & $\overline{3}$ & $\begin{array}{l}\text { toke remplace } \\
\text { took }\end{array}$ \\
\hline 14 - Erreur d'emprunt & 8 & 2 & $\begin{array}{l}\text { ported remplace } \\
\text { carried }\end{array}$ \\
\hline 15 - Erreur d'accord & 7 & 2 & $\begin{array}{l}\text { we was remplace } \\
\text { we were }\end{array}$ \\
\hline $\begin{array}{l}16 \text { - Erreur d'usage : past } \\
\text { perfect remplace prétérit }\end{array}$ & 7 & 2 & $\begin{array}{l}\text { had fallen } \\
\text { remplace fell }\end{array}$ \\
\hline $\begin{array}{l}17 \text { - Erreur de forme } \\
\text { négative }\end{array}$ & 5 & 1 & $\begin{array}{l}\text { don't knew } \\
\text { rempl ace didn't } \\
\text { know }\end{array}$ \\
\hline $\begin{array}{l}18 \text { - Erreur d'usage : } \\
\text { présent progressif } \\
\text { remplace prétérit }\end{array}$ & 5 & 1 & $\begin{array}{l}\text { am going } \\
\text { remplace went }\end{array}$ \\
\hline Total & 334 & 100 & \\
\hline
\end{tabular}

Tableak 2 - Distribution en fréquence des types d'erreurs. 
sans doute en référence à il s'est levé. En bref, il existerait une centration sur la forme des verbes français pour sélectionner la forme des verbes anglais. Cette centration s'effectuerait au détriment des conditions d'usage du prétérit anglais.

\begin{tabular}{|ccc|cc|}
\hline Niveau & \multicolumn{2}{c|}{$\begin{array}{c}\text { Correspondance } \\
\text { avec } \\
\text { l'auxiliaire français }\end{array}$} & $\begin{array}{c}\text { Non correspondance avec } \\
\text { l'auxiliaire français }\end{array}$ \\
& occurrence & $\%$ & occurrence & $\%$ \\
$4^{\mathrm{e}}$ & 17 & 47.22 & 19 & 52.77 \\
$\mathbf{3}^{\mathrm{e}}$ & 16 & 84.21 & 3 & 15.78 \\
$\mathbf{2}^{\mathrm{e}}$ & 21 & 80.76 & 5 & 19.23 \\
$\mathbf{1}^{\text {re }}$ & 7 & 100 & 0 & 0 \\
Totaux & 61 & 69.31 & 27 & 30.68 \\
\hline
\end{tabular}

Tableau 3 - Occurrences et pourcentage des correspondances des auxiliaires anglais avec les auxiliaires français dans le cas des erreurs be conjugué et have conjugué.

\section{Étude expérimentale}

Pour prouver l'existence d'une telle centration sur les temps composés du français et plus particulièrement sur le passé composé, nous avons demandé à trente sujets de classe de quatrième du collège de traduire des phrases comportant des formes verbales passées du français de deux types: certaines au passé composé, et d'autres au passé simple. Nous avons testé plusieurs types de verbes anglais: des verbes réguliers et des verbes irréguliers, certains fréquents et d'autres rares ${ }^{2}$. Ces verbes sont listés dans le Tableau 4 (voir p. suiv.).

Ces 16 verbes étaient insérés dans 32 phrases en français qui se présentaient comme dans l'exemple suivant:

2. À défaut d'une description des fréquences dans l'environnement de la classe de langue, nous avons utilisé les valeurs utilisées par Rumelhart et McClelland (1987) élaborées à partir du corpus de Brown, établi par Kucera et Francis (1964), et consistant en un million de mots provenant de 15 catégories différentes de textes. 
En 1998, la France a joué............ (PLAY) la coupe du monde de foot contre le Brésil.

En 1911, les rugbymen français jouèrent........... (PLAY) leur premier tournoi des cinq nations.

\begin{tabular}{|c|c|c|c|c|c|c|}
\hline & \multicolumn{3}{|c|}{$\begin{array}{c}\text { VERBES } \\
\text { REGULIERS }\end{array}$} & \multicolumn{3}{|c|}{$\begin{array}{c}\text { VERBES } \\
\text { IRREGULIERS }\end{array}$} \\
\hline & $\mathrm{BV}$ & $\begin{array}{l}\text { Forme } \\
\text { prétérit }\end{array}$ & $\begin{array}{l}\text { Traduc- } \\
\text { tion }\end{array}$ & BV & $\begin{array}{l}\text { Forme } \\
\text { prétérit }\end{array}$ & $\begin{array}{l}\text { Traduc- } \\
\text { tion }\end{array}$ \\
\hline \multirow{4}{*}{$\begin{array}{c}\text { Verbes } \\
\text { fréquents }\end{array}$} & Play & Played & Jouer & Go & Went & Aller \\
\hline & Start & Started & $\begin{array}{l}\text { Commen- } \\
\text { cer }\end{array}$ & Give & Gave & Donner \\
\hline & Walk & Walked & Marcher & Make & Made & Faire \\
\hline & Love & Loved & Aimer & Have & Had & Avoir \\
\hline \multirow{4}{*}{$\begin{array}{l}\text { Verbes } \\
\text { rares }\end{array}$} & Mail & Mailed & $\begin{array}{l}\text { Envoyer } \\
\text { une lettre }\end{array}$ & Bid & $\begin{array}{l}\text { Bade / } \\
\text { Bid }\end{array}$ & $\begin{array}{l}\text { Offrir un } \\
\text { prix }\end{array}$ \\
\hline & Hug & Hugged & $\begin{array}{l}\text { Serrer } \\
\text { dans ses } \\
\text { bras }\end{array}$ & Creep & Crept & Grimper \\
\hline & Guard & Guarded & Surveiller & Breed & Bred & Elever \\
\hline & Carp & Carped & Maugréer & Dig & Dug & Creuser \\
\hline
\end{tabular}

Tableau 4 - Verbes utilisés dans l'expérimentation.

Une phrase rédigée en français comportait une forme verbale, simple (passé simple) ou composée (passé composé) selon le cas, soulignée et accompagnée de la base du verbe anglais entre parenthèses afin que l'effort de traduction se 
concentre essentiellement sur le choix d'un paradigme verbal de l'anglais. Les phrases étaient présentées sur un carnet dans un ordre aléatoire, à raison d'une seule phrase par page, afin de limiter les interférences entre réponses successives. La consigne était de traduire en anglais le verbe souligné et visait à orienter l'attention des sujets sur les formes verbales.

En constituant les phrases, nous avons pris garde de n'utiliser que des passés composés à valeur temporelle de passé, c'est-à-dire se traduisant nécessairement par un prétérit simple anglais. De plus, pour éviter toute ambiguïté, nous avons employé dans chaque phrase des indicateurs indiquant le décrochage par rapport au moment de l'énonciation et exigeant l'emploi du prétérit simple.

Les résultats montrent que globalement le remplacement par une forme composée est significativement plus fréquent quand la source est composée, c'est-à-dire quand la forme verbale française est au passé composé. Lorsque le verbe source en français est au passé composé, en moyenne 7,9 formes traduites sur un maximum de 16 sont des formes composées (soit $49,3 \%$ ). Si le verbe source en français est au passé simple, ce score chute à 1,35 (soit $8,4 \%$ ).

Nous avons ensuite observé si cette différence persistait dans chaque catégorie de formes verbales (Tableau 5). Pour les quatre points de comparaison du tableau, le test de Wilcoxon indique que la présence d'une forme composée dans le

\begin{tabular}{|c|c|c|c|c|}
\hline \multirow[t]{2}{*}{ Maximum $=4$} & \multicolumn{2}{|c|}{ VERBES REGULIERS } & \multicolumn{2}{|c|}{ VERBES IRREGUL IERS } \\
\hline & Source simple & $\begin{array}{c}\text { Source } \\
\text { composée }\end{array}$ & Source simple & $\begin{array}{c}\text { Source } \\
\text { camposée }\end{array}$ \\
\hline $\begin{array}{c}\text { Verbes } \\
\text { fréquents }\end{array}$ & $(0.57 \%)$ & $\begin{array}{c}2.00 \\
(1.414)\end{array}$ & $\begin{array}{c}0.10 \\
(0.308)\end{array}$ & $\begin{array}{c}1.70 \\
(1.380)\end{array}$ \\
\hline Verbes rares & $\begin{array}{l}0.55 \\
(0.887)\end{array}$ & $\begin{array}{c}2.05 \\
(1.761)\end{array}$ & $(0.80$. & $\begin{array}{c}2.15 \\
(1.785)\end{array}$ \\
\hline
\end{tabular}

Tableau 5 - Traduction par des formes simples ou des formes composées, en fonction de la source (temps simple vs composé en français), de la régularité et de la fréquence des verbes anglais (moyenne et écart type entre parenthèses). 
verbe source français induit la traduction de ce verbe par une forme composée anglaise, quelles que soient la régularité et la fréquence du verbe anglais. Ces résultats valident l'hypothèse selon laquelle les sujets forment les verbes anglais par référence aux formes composées du français, notamment au passé composé.

Une analyse détaillée des erreurs produites lors de l'expérimentation montre par ailleurs que le nombre de régularisations est nettement supérieur à ce que nous avons constaté dans les textes narratifs. Dans 1'étude exploratoire sur les productions d'écrits, nous avions trouvé au total 14 erreurs de régularisation sur 1006 verbes irréguliers d'où un pourcentage de régularisation de $0,20 \%$. Dans l'expérimentation, le taux global de régularisation est de $25,3 \%$ soit 126 fois plus que dans le corpus. Cette différence suggère que l'expérimentation favorise ces régularisations en imposant la traduction de formes verbales irrégulières rares.

\section{Discussion}

Nous constatons que l'acquisition du prétérit anglais par des apprenants français diffère de son acquisition par les jeunes Anglais. En effet, certains des résultats obtenus par 1'analyse du corpus et par le protocole expérimental ne correspondent pas aux points forts du scénario développemental élaboré par Terezinha Nunes et ses collègues (1997).

Premièrement, les erreurs de régularisation, fréquentes chez les jeunes anglophones, sont quasi inexistantes dans les textes des jeunes Français mais nombreuses dans l'expérimentation. La confrontation entre un recueil contrôlé des données par traduction et un recueil plus spontané par narration écrite indique que la régularisation est un processus disponible chez les apprenants français, mais peu exploité en production libre de textes, sans doute du fait de l'évitement des verbes irréguliers peu ou pas connus.

Deuxièmement, nous observons un phénomène massif, dont l'origine n'a jamais été établie empiriquement dans les études sur l'apprentissage du prétérit anglais par les jeunes Anglais : le remplacement d'une forme simple par une 
forme composée. L'analyse du corpus indique également que l'auxiliaire impliqué dans les erreurs que nous avons nommées «be conjugué » et «have conjugué » correspond dans $69 \%$ des cas à l'auxiliaire français.

Nous interpréterons ce classique phénomène de transfert dans les termes d'une focalisation de la conscience morphosyntaxique des sujets français sur la forme au détriment de l'usage. Les apprenants chercheraient à reproduire la séquence "auxiliaire + participe passé " du français et négligeraient les conditions d'usages des temps de l'anglais. On peut supposer que cette centration sur la forme au détriment de l'usage provient de l'absence d'un enseignement de l'aspect dans la grammaire scolaire du français. Plus précisément, la distinction entre le passé composé à valeur d'accompli du présent, qui se traduit par un present perfect anglais, et le passé composé à valeur aoristique, qui se traduit par un prétérit anglais, ne fait pas l'objet d'un enseignement systématique à l'école primaire et au collège. Démunis des bases métagrammaticales leur permettant de comprendre les conditions d'usage des temps de l'anglais, les jeunes Français utiliseraient la forme composée ou la forme simple du français comme unique repère $\mathrm{Ce}$ serait donc bien un défaut de la conscience morphosyntaxique dans la langue maternelle qui provoquerait l'erreur dans la langue étrangère.

Dans le domaine de la langue maternelle, on connait depuis longtemps l'impact de la conscience métalinguistique sur les apprentissages scolaires de l'écrit (Gombert, 1990). Plusieurs études ont démontré également que les connaissances métalinguistiques en langue maternelle sont étroitement liées à la réussite de l'apprentissage de la langue étrangère. Ce résultat est établi pour l'apprentissage de l'hébreu par des enfants anglais et l'apprentissage de l'anglais par des enfants portugais, même si les langues en jeu ont des structures différentes (Bindman, 2003; Castro, 2003). Ce transfert ne s'effectuerait pas seulement de la langue maternelle vers la langue étrangère. En effet, les principes grammaticaux acquis en langue étrangère peuvent apporter une meilleure compréhension aux enfants qui sont en train de développer leur conscience grammaticale en langue maternelle. Ceci peut s'avérer vrai pour des aspects grammaticaux qui sont marqués 
plus clairement en langue étrangère qu'en langue maternelle et qui bénéficient d'une instruction explicite.

Dès lors, nous rejoignons les résultats précurseurs et les positions défendues par Louise Dabène (1983) dans un cadre didactique: un travail métalinguistique sur la grammaire de la langue maternelle en situation d'apprentissage scolaire doit contribuer à une meilleure maitrise du système de la langue étrangère.

\section{Références bibliographiques}

BINDMAN, M. (2003): Grammatical awareness across languages and the role of social context: Evidence from English and Hebrew, in T. Nunes et P. Bryant (Eds.). Handbook of children's literacy, Dordrecht, Kluwer.

BRISSAUD, C. et CHEVROT, J.-P. (2000): Acquisition de la morphographie entre 10 et 15 ans : le cas du pluriel des formes verbales en /E/, Verbum 22 (4), 245-439.

CASTRO, M.-I. (2003): Grammatical awareness and foreign language learning, MA. Oxford Brookes University.

DABÈNE, L. (1983). Le métalangage: un point de rencontre obligé entre enseignants de langue maternelle et de langue étrangère, Le français dans le monde 177, 45-49.

GOMBERT, J.-É. Le développement métalinguistique. P.U.F., Paris.

JAFFRÉ, J.-P. (1998): L'orthographe du français, une exception?, Le Français Aujourd'hui, 122, 45-53.

KUCERA, H. et FRANCIS, W. (1964): Computational Analysis of Present-day American English, Providence, RI : Brown University Press.

LAPAIRE, J.-R et ROTGE, W. (1991): Linguistique et grammaire de l'anglais, Toulouse, Presses Universitaires du Mirail.

LARREYA, P. et RIVIERE, C. (1991): Grammaire explicative de l'anglais, Paris, Longman France.

LUCCI, V. et MILLET, A. (1994): L'orthographe de tous les jours, enquête sur les pratiques orthographiques des Français, Paris, Editions Champion.

NUNES, T., BRYANT, P. et BINDMAN, M. (1997): Orthographe et 
grammaire: the necsed move, in C. Perfetti, M. Fayol et L. Rieben (Eds.), Des orthographes et leur acquisition, Lausanne, Delachaux et Niestlé, 101-123.

RUMELHART, D.-E. et MCCLELLAND, J.-L. (1987): On learning the past tenses of English verbs, in J.-L. McClelland, D.-E. Rumelhart, et PDP Research Group (Eds), Parallel distributed processing: explorations in the microstructure of cognition, vol. II, 216-271, Cambridge, MA: Bradford/MIT Press.

THÉVENIN, M.-G., TOTEREAU, C. et FAYOL, M. (1997): Acquisition de la morphologie du nombre à l'écrit en français, in C. Perfetti, M. Fayol et L. Rieben (Eds.), Des orthographes et leur acquisition, Lausanne, Delachaux \& Niestlé, 147-165. 